Article

\title{
Analysis of Electromagnetic Waves Attenuation for Underwater Localization in Structured Environments
}

\author{
Daegil Park ${ }^{1}$, Kyungmin Kwak ${ }^{2}$, Wan Kyun Chung ${ }^{3}$ and Jinhyun Kim ${ }^{4 *}$ \\ Korea Institute of Robot and Convergence(KIRO); daegilpark@kiro.re.kr \\ CJ Logistics; kyungmin.kwak@cj.net \\ Pohang University of Science and Technology(POSTECH); wkchung@ postech.ac.kr \\ 4 Seoul National University of Science and Technolog(SeoulTECH); jinhyun@ seoultech.ac.kr \\ * Correspondence: jinhyun@ seoultech.ac.kr; Tel.: +82-070-8252-6318
}

\begin{abstract}
In this paper analyses the characteristic of EM waves propagation in structured environment to identify the signal interference by the structure, and suggests the EM waves attenuation model considering the distance and penetration loss by the structure. The range sensor based on electromagnetic(EM) waves attenuation along to the distance showed the precise distance estimation with high resolution depending on the distance. However, it is hard to use in structured environments due to the lack of consideration of the EM waves attenuation characteristics in the structured underwater environment. In this paper, EM waves propagation characteristic and signal interference effects by the structures were analyzed, and the EM waves distance-attenuation model in structured environment was suggested with sensor installation guideline. The EM waves propagation characteristics and proposed sensor model were verified by the several experiments, and the localization result in structured environment showed the more reliable performance.
\end{abstract}

Keywords: Underwater range sensor; Underwater localization; sensor network; Received signal strength

\section{Introduction}

With increasing underwater infrastructures such as offshore plant and offshore wind power, many studies about unmanned underwater vehicles (UUV) are recently started to maintain underwater structures. There have been many researched for the underwater localization, which is essential for the UUV perception in underwater environment[1,2].

For all that, the sensor for underwater localization is conventionally limited to acoustic sensors, since the sonar sensor has a long range and reliable operation underwater environments. However, sonar does not guarantee a performance in complicated structured environments due to the multi-path effect and diffraction scattering. Moreover, by increasing the underwater structures, many applications need precise position estimation in complicated structured environments such as offshore plant and docking structure [3-7]. Therefore, an alternative sensor to use in underwater structure environments is required.

To overcome the aforementioned problems, we suggest a method of estimating locations using the electromagnetic(EM) waves attenuation characteristics along the distance. The proposed sensor showed very precise distance estimation with high resolution depending on the distance. Also, EM waves propagate much faster than do sound waves, so use of EM can achieve a high sampling rate; this characteristic can be exploited for use in dynamic object tracking[8-11].

However, it is difficult to use the proposed localization system in real applications due to the lack of consideration of the EM waves attenuation characteristics in the structured underwater environment. The previous works were only considered the received signal strength(RSS) of EM waves in lossy medium, and verified accuracy in an ideal condition. But most underwater positioning applications and sensor installation conditions are focused on the complex and structured environments. Therefore, the analysis of EM waves propagation near the some objects are needed to use the EM waves attenuation sensor near the structured environments.

In this paper, EM waves propagation characteristic and signal interference effects by the structures were analyzed. The Fresnel zone and near-field were considered as the distortion criterion, and were verified the 


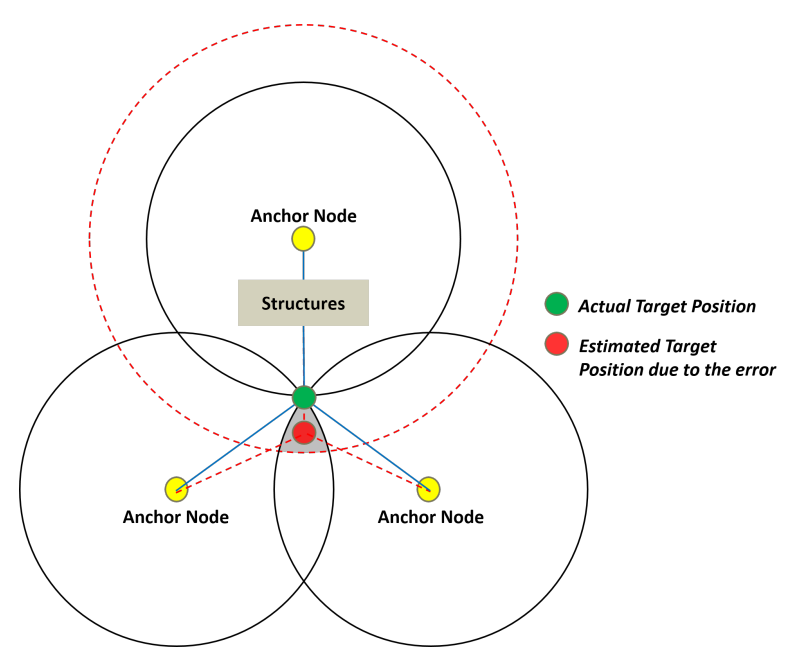

Figure 1. Conceptional diagram of biased localization result caused by some structure. The structure brings about additional EM waves attenuation and it functions as additional distance gap between nodes.

several interferences in water medium by the feasibility tests. Based on these analyses and experiments, the EM waves distance-attenuation model in structured environment was suggested with sensor installation guideline.

This paper is organized as follows. Section II introduces the previous works for underwater range sensor model in ideal condition and model parameter estimation scheme in infrastructure-based localization system. Then, the theoretical analysis of EM waves interference near objects derived in section III. The underwater EM waves propagation characteristics is verified with several experiments in section IV. The signal loss due to an object penetration is considered as the attenuation attenuation model in structured environments in section V. Section VI shows the proposed sensor model performance by comparing the estimated sensor parameter and 2D localization in structure environment. Finally, section VII presents the summary, conclusion, and outline for future work.

\section{Derivation of Underwater Sensor Model and Model Parameter Estimation}

\subsection{Underwater range sensor model}

An EM waves attenuation according to the distance is affected by antenna shapes, frequency and medium properties. Fortunately, the medium attenuation can be leaved out of consideration in air condition because it is very small enough to omit. So we can only consider the energy diffusion as a function of distance by using Friis-Shelkunoff formula[12]. However, in other mediums such as water or oil with large attenuation, the Friis-Shelkunoff formula is not enough to calculate the distance. For this reason, an additional formula that accounts for the media properties, attenuation and absorption, is needed. Therefore we proposed the novel underwater sensor model combining both energy diffusion and energy absorption by the medium.

\subsubsection{Friis-Shelkunoff Formula}

The Friis-Shelkunoff formula is a basic antenna theory which calculates the separation distance $R$ between a transmission antenna with $G_{T}$ and a receiving antenna with gain $G_{R}$ for an EM waves with frequency $f$. Due to the low attenuation of EM waves in the air, the attenuation is assumed to be zero.

If the antennas are aligned and the distance $R$ exceeds the near field distance $\left(R_{n}=\frac{L^{2}}{\lambda}\right.$, where $L$ is the maximum dimension of the distance), the relationship between the received signal power $P_{R}$ and distance $R$ is given by the Friis-Shelkunoff formula:

$$
P_{R}=\frac{P_{T} G_{T} G_{R} \lambda^{2}}{(4 \pi R)^{2}}[\mathrm{~mW}]
$$




\subsubsection{Attenuation Constant}

Generally, the power attenuation by medium as a function of distance can be expressed by an attenuation constant of the plane wave equation [13].

In the plane wave equation, $P_{R}$ is described by $P_{T}, R$, and the attenuation coefficient $\alpha$ as

$$
P_{R}=P_{T} e^{-2 \alpha R}[\mathrm{~mW}]
$$

where, $\alpha$ is the real part of the propagation constant $\gamma$.

\subsubsection{Propagation Formula for a Lossy Medium}

To acquire the RSS for a specific medium for a given distance, we should consider the properties of both the antenna and medium simultaneously. Assuming that the antennas radiate a wave which diverges approximately spherically in the far field area, and propagate with the plane wave in the medium, we estimate the combined formula for EM waves.

By considering both the transmission power and the properties of the EM waves, we combine the attenuation constant, Eq. (2) and the Friis-Shelkunoff formula, Eq. (1), resulting in

$$
P_{R}=\frac{P_{T} G_{T} G_{R} \lambda^{2}}{(4 \pi R)^{2}} e^{-2 R \alpha}[\mathrm{mW}]
$$

The above equation is rearranged and simplified as follows:

$$
P_{R}=\frac{e^{-2 \alpha R}}{R^{2}} \times c
$$

where $c$ is the constant term which is not influenced by $R$. To change the unit to $\mathrm{dB}$, we take the logarithm of both sides:

$$
10 \log _{10} P_{R}=-20 \log _{10} R-20 \alpha R \log _{10} e+10 \log _{10} c
$$

By replacing the transfer power $10 \log _{10} P_{R}, 10 \log _{10} P_{T}, 10 \log _{10} c$ with the new log-scale constant $R S S$ (or $S_{R}$ ), $S_{T}$ and $C$, the following $R S S$ equation with distance can be modeled as follows:

$$
\begin{gathered}
R S S=-20 \log _{10} R-20 R \alpha \log _{10} e+C[\mathrm{dBm}] \\
C=20 \log _{10} \frac{\lambda}{4 \pi}+10 \log _{10} G_{T}+S_{T}+10 \log _{10} G_{R}
\end{gathered}
$$

As shown in Eq. (6), the RSS of the underwater EM waves is calculated as the sum of logarithmic and linear functions of $R$ and the constant term [8,11].

\subsection{Sensor Model Calibration as Parameter Estimation}

Generally, it is difficult to exactly calculate the constant term $C$ and the attenuation constant $\alpha$ because each parameter is affected by many environmental conditions (antenna im-pedance mismatch caused by the medium, environmental effects not modeled, noise, etc.) and medium properties (conductivity, permeability, permittivity). Moreover, it is necessary to measure the parameters of the sensor periodically, because the ratio of EM waves attenuation can continuously change. However, it is not only very difficult to periodically check the many parameters but also additional equipments are necessary for the task, further complicating the process. Fortunately, if the localization system has anchor node more than three, we can conduct a parameter estimation using the characteristics of the localization system.

Almost all RSS-based localization systems rely heavily on anchor nodes on an infrastructure environment, as shown in Fig. 2. The anchor nodes $(1,2, \cdots, i, j)$ provide known positions and distances. By plugging in 


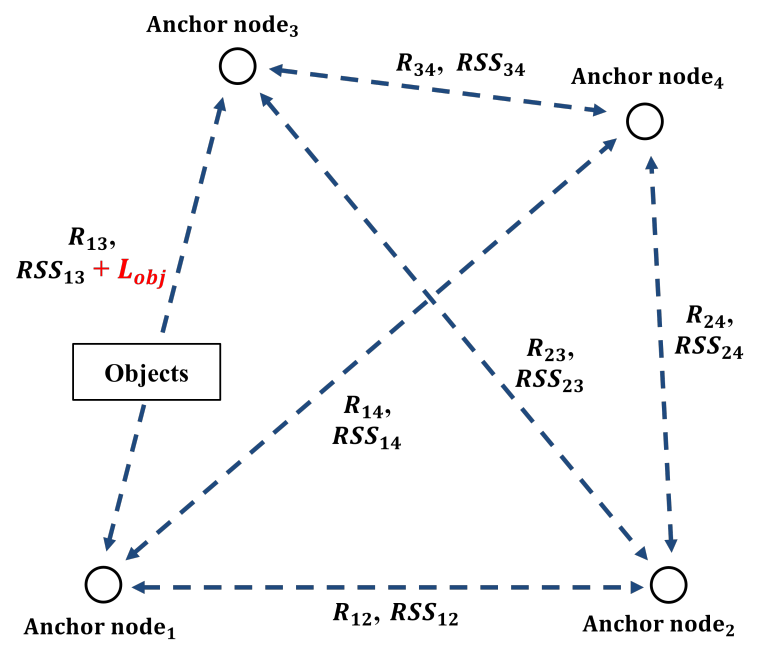

Figure 2. Conceptual diagram of parameter estimation using the anchor node information. Using the estimated value $R S S_{n}$ at the reference distance $R_{n}$, a user can determine $C$ and $\alpha$. However, if structure exists between anchor nodes such as anchor node ${ }_{1}$ and anchor node ${ }_{3}$, it bring about the uncertainty of parameter estimation due to an additional loss $\left(L_{o b j}\right)$.

our RSS estimates $\left(R S S_{12}, R S S_{13}, \cdots, R S S_{i j}\right)$ and their known distances $\left(R_{12}, R_{13}, \cdots, R_{i j}\right)$ into Eq. (6), we can estimate the parameter $\alpha$ and $C$ estimation using input / output mapping as the follows:

$$
\begin{gathered}
R S S_{12}=-20 \log _{10} R_{12}-20 R_{12} \alpha \log _{10} e+C \\
R S S_{13}=-20 \log _{10} R_{13}-20 R_{13} \alpha \log _{10} e+C \\
\vdots \\
R S S_{i j}=-20 \log _{10} R_{i j}-20 R_{i j} \alpha \log _{10} e+C
\end{gathered}
$$

Therefore, we can easily approximate the attenuation factor $\alpha$ and the constant term $C$ using a least square method.

However, if structure exists between anchor nodes during the parameter estimation, it bring about the uncertainty of parameter estimation results. Therefore, sensor network must be maintained an open space condition during calibration scheme.

\section{Analysis of EM Waves Interference Near Objects}

Generally, the propagation characteristics of EM waves are considered as radiating into an unbounded medium. However, the presence of a structure, especially when it is near the radiating element, can significantly alter the overall radiation properties of the antenna system. In fact, in most cases, structures exist in the propagation path of EM waves (even in the absence of anything else, is the ground). Therefore, it is very important to understand the environmental influence between the paths of electromagnetic waves propagation.

However, it is very difficult problem estimate the energy loss by these interferences. The interference can be classified into penetration, deflection and diffraction, which are affected by the object's characteristics such as radius, thickness, material property, shape and so on. So, if a EM waves energy loss model can be offered with all of these characteristics, it is a convenient way to estimate an additional attenuation power. However, that model not only is infeasible approach that measures all characteristics, it has also large computation loads. Moreover, it is hard to measure the variable factors depend on the mobile node conditions such as incidence angle and surface roughness. Therefore, it is important to minimize the effects of the objects thorough EM waves propagation analysis and to design the additional loss model for most important (most influential and measurable) interference. 


\subsection{Fresnel zone in water condition}

The EM waves propagation may be interrupted by objects when the they exists between transceivers, and it causes a phase shift of the electromagnetic waves. These phase shift effect can not be measured exactly. Alternatively, the effects of interference can be checked using Fresnel zone which be used to analyze interference by objects near the path of EM waves.

The general equation for calculating the Fresnel zone radius is the following [14]:

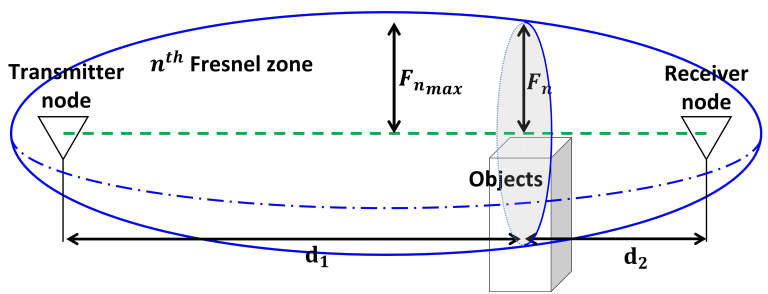

Figure 3. Conceptional diagram of Fresnel zone. The Fresnel zone means the EM Waves interference region by some objects between transmitter and receiver.

$$
F_{n}=\sqrt{\frac{n \lambda d_{1} d_{2}}{d_{1}+d_{2}}}
$$

where $\lambda$ is the wavelength of EM waves, $n$ is the order of Fresnel zone, and $d_{1}, d_{2}$ are the distance between an object and node in Fig. 3 .

where $\lambda$ is the wavelength of EM waves, $n$ is the order of Fresnel zone, and $d_{1}, d_{2}$ are the distance between an object and node in Fig. 3. EM waves interference by objects decreases dramatically as the order of Fresnel zone increases. The high order Fresnel zone must be kept largely free from obstructions to avoid interfering with the radio reception. In this case, the propagation of electromagnetic waves is hardly affected by the object, and it can be assumed that the EM waves is propagated in the open space. If some parts of Fresnel zone encounter with the objects, the EM waves can be occurred the multi-path effect, and it is hard to estimate due to the changeable factors depends on conditions. And if the whole of Fresnel zone belong to the objects, almost EM waves signal propagates to the receiving antenna by penetrating objects with signal loss. In the water condition, the Fresnel zone in underwater has smaller size than air, because the wavelength of EM waves becomes smaller in denser medium $\left(\lambda_{\text {water }} \approx \lambda_{\text {air }} / 8.8\right.$, at $\left.420 \mathrm{Mhz}, 25^{\circ} \mathrm{C}\right)$.

\subsection{Near field region}

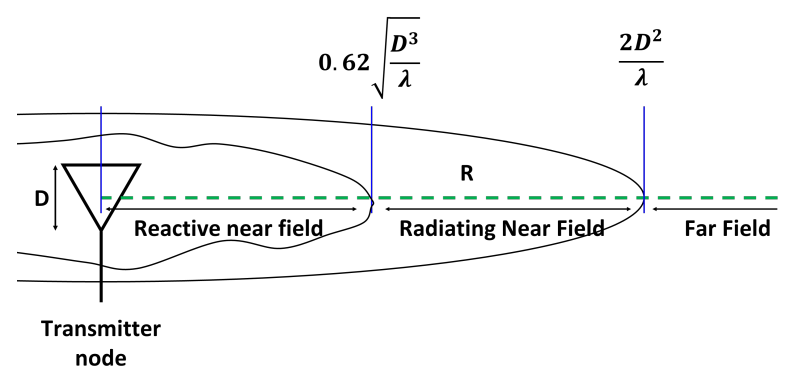

Figure 4. Conceptional diagram of near field region. EM waves suffers the irregular radiation pattern near the transmitter due to the phase gap between E-field and H-field. Although the objects belong to the near-field of transmitter, the fields still die off as $1 / R$, the power density dies off as $1 / R^{2}$.

Another factor influencing the propagation characteristics of EM waves is the distance from the transmitter (Fig. 4). Near the transmitter, the radiation pattern of EM waves does not change shape with distance. In the 
immediate vicinity of the transmitter, the EM waves has the reactive near field, which means the E- and H- fields are out of phase of 90 degrees to each other. The equation for calculating the reactive near field is the following [15]:

$$
R<0.62 \sqrt{\frac{D^{3}}{\lambda}} .
$$

where, $D$ denotes the antenna of maximum linear dimension.

And the radiating near field is the region between the near and far fields. In this region, the reactive fields are not dominate. However, here the shape of the radiation pattern may vary appreciably with distance. The equation for calculating the radiating near field is the following:

$$
0.62 \sqrt{\frac{D^{3}}{\lambda}}<R<\frac{2 D^{2}}{\lambda}
$$

Although the objects belong to the near-field of transmitter, the fields still die off as $1 / R$, the power density dies off as $1 / R^{2}$.

\section{EM Waves Propagation Experiments Near the Objects}

Several experiments were conducted in the water basin to verify the EM waves propagation characteristic near the objects. The experiments were (1) the antenna input impedance measurement near the objects to check the near-field effects, (2) the RSS measurement near the objects to check the EM waves multi-path effect according to the Fresnel zone, and (3) the RSS measurement to check the EM waves penetration loss characteristic in side of Fresnel zone.

\subsection{Common experimental environment}

Table 1. Experimental Environment Constants

\begin{tabular}{ccc}
\hline & Property (symbol) & Values [unit] \\
\hline Conductivity $(\sigma)$ & $0.075[\mathrm{~S} / \mathrm{m}]$ \\
Freshwater & Permeability $(\mu)$ & $1.2566 \times 10^{-6}[\mathrm{H} / \mathrm{m}]$ \\
& Permittivity $(\varepsilon)$ & $7.2797 \times 10^{-10}[\mathrm{~F} / \mathrm{m}]$ \\
& Wavelength at 420Mhz $(f)$ & $0.0811[\mathrm{~m}]$ \\
& Refraction index $(n)$ & 8.8 \\
\hline \multirow{3}{*}{ Antenna } & Antenna gain $\left(G_{T}, G_{R}\right)$ & $3[\mathrm{dBi}]$ \\
& Maximum linear dimension $(\mathrm{D})$ & $0.315[\mathrm{~m}]$ \\
& Transmitting Power $\left(S_{T}\right)$ & $10[\mathrm{dBm}]$ \\
& Operation frequency $(f)$ & $420[\mathrm{MHz}]$ \\
\hline
\end{tabular}

To check the EM waves interferences, we set up the experiment in underwater test facility in Korea Institute of RObot and convergence (KIRO). The test tank is $12 \mathrm{~m}$ long, $8 \mathrm{~m}$ wide, and $6 \mathrm{~m}$ deep. To prevent EM wave reflection, the antennas were separated $1.5 \mathrm{~m}$ away from the wall using an aluminum experimental guide rail and were submerged $1.5 \mathrm{~m}$. The antennas used in the experiment were dipole antennas with an antenna gain of $3 \mathrm{dBi}$, and the transmitting and receiving antennas were installed (Table 1). To ensure proper alignment between the antennas, antenna frames were used. The distance between the antennas was measured as the distance between the antenna frames using a tapeline and a laser range finder. The medium inside the basin is assumed to be fresh 
water. EM wave generation and signal reception were carried out with a National Instruments signal generator (NI5660SA) and signal analyzer (NI5670SG). The transmitting power was set to $10 \mathrm{~mW}(10 \mathrm{dBm})$ with $420 \mathrm{MHz}$ frequency.

\subsection{Input impedance influence near objects}

4.2.1. Condition and procedure

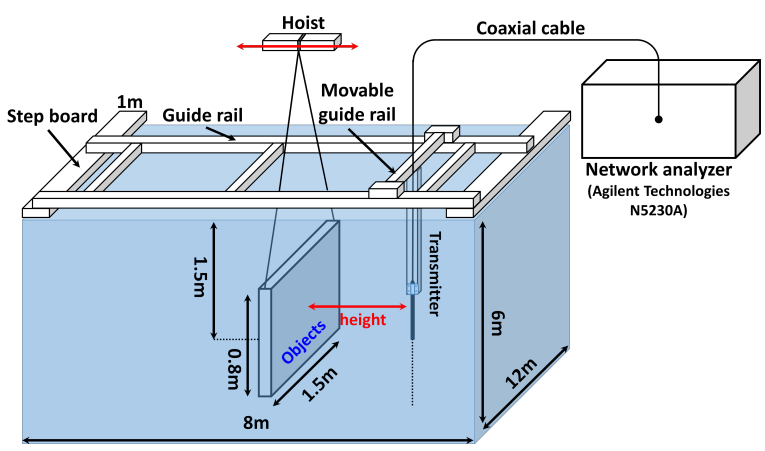

Figure 5. Schematic diagram of input impedance experiment near the objects.

The experimental environment was configured to check the input impedance influence in the vicinity of objects, as shown in Fig. 5. The network analyzer (Agilent Technologies N5230A) measured the input impedance of transmitter antenna at $420 \mathrm{Mhz}$ according to the distance(height) between transmitter and object. The height between the antenna and object was incrementally increased by $0.01 \mathrm{~m}$, starting at $0.03 \mathrm{~m}$ up to $0.5 \mathrm{~m}$. A steel plate and wood plate (dielectric constant $\approx 2$ ) were used to check the effects of an object.

4.2.2. Results

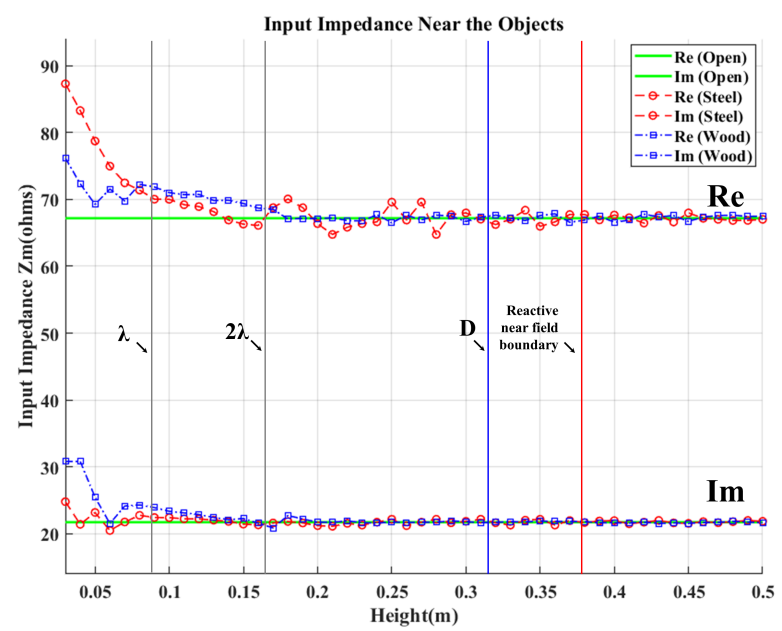

Figure 6. The input impedance versus height.

The input impedance versus distance data was collected, and the results are shown in Fig. 6. The input impedance value was clearly increased when the height was less than $2 \lambda$. However, when distance between antenna and object was bigger than $2 \lambda$ and maximum linear dimension $(D)$, the input impedance value had similar to an input impedance in open environment despite it belong to the reactive near field. It estimated that water has different near field characteristics with the air because it is lossy medium. Based on this experiment, the transmitter installation is recommended to keep a distance at least $D$ and $2 \lambda$ from the structures to prevent the antenna impedance mismatching and near field effect. 


\subsection{EM waves interference in the Fresnel zone}

4.3.1. Condition and procedure

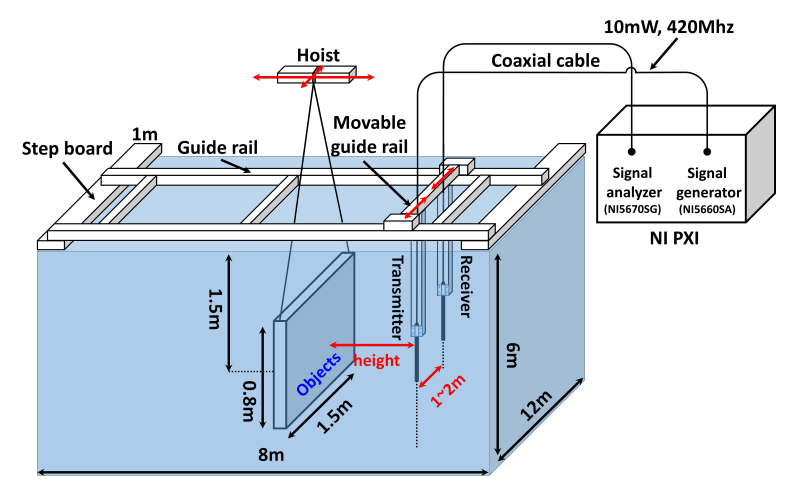

Figure 7. Schematic diagram of EM waves interference experiment in the Fresnel zone.

The experimental environment was configured to check the EM waves interference in the Fresnel zone, as shown in Fig. 7. The signal analyzer measured the RSS of EM waves at 420Mhz according to the distance and height. The height between the antenna and object was incrementally increased by $0.025 \mathrm{~m}$, starting at $0.1 \mathrm{~m}$ up to $0.55 \mathrm{~m}$. This experiment was repeated three times along to the change of distance between transmitter and receiver $(\mathrm{R}=1 \mathrm{~m}, 1.5 \mathrm{~m}$, and $2 \mathrm{~m})$. A steel plate and wood plate were used to check the effects of an object.

4.3.2. Results

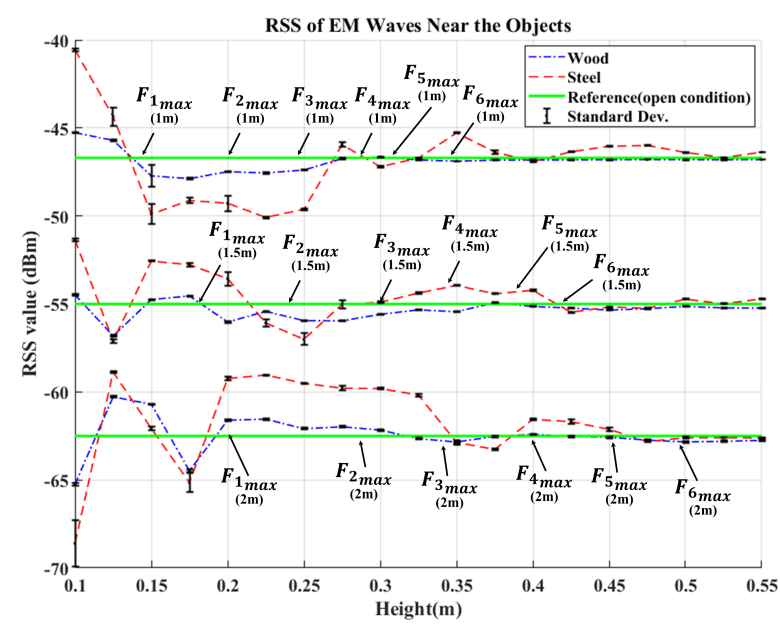

Figure 8. The RSS of EM waves versus hight and distance.

The RSS value versus distance and height data was collected, and the results are shown in Fig. 8 . The antennas go away from the object, the RSS value converged to the RSS value in the open environment. The change of RSS value showed more prominent as the distance between the two antennas increased, because the radius of the Fresnel zone increases as the distance between two antennas increases. The RSS values had large unexpected fluctuation with big standard derivation when objects belong to the 1 st 2 nd Fresnel zone. It estimated the multi-path effects of EM waves. However, there had a few RSS change when the height is greater than radius of 4th Fresnel zone. Based on this experiment, the straight line between antennas are recommended to keep a distance at least 4th Fresnel zone radius from the structures to prevent the unexpected RSS change caused by multi-path effect. 
4.4.1. Condition and procedure

\subsection{Penetration loss by objects}

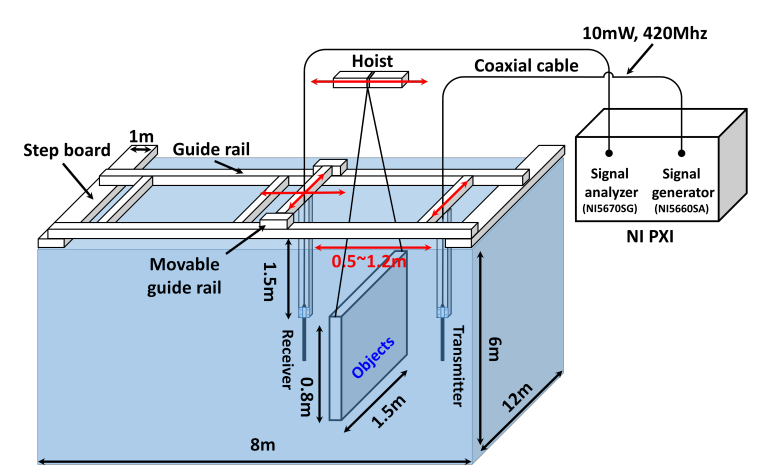

Figure 9. Schematic diagram of experiments for EM waves penetration effect in the Fresnel zone.

The experimental environment was configured to check the EM waves interference when whole of 4th Fresnel zone belong to the objects, as shown in Fig. 9. The signal analyzer measured the RSS of EM waves at 420Mhz depends on three experiments: (1) change the relative position between object and antennas in direction of the EM waves propagation (Fig. 10(a)). (2) Change the distance between antennas at fixed object position (Fig. 10(b)).

4.4.2. Results
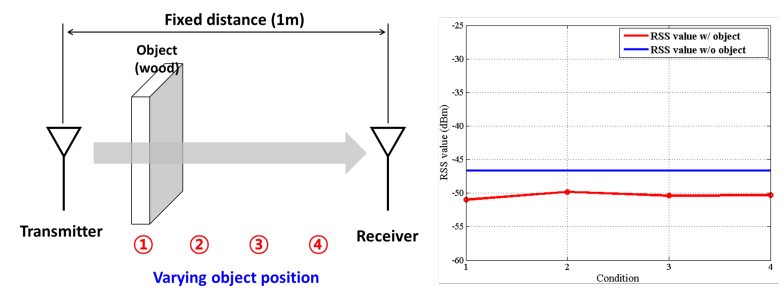

(a) Case 1: Fixed distance with varying relative position (in direction of propagation)

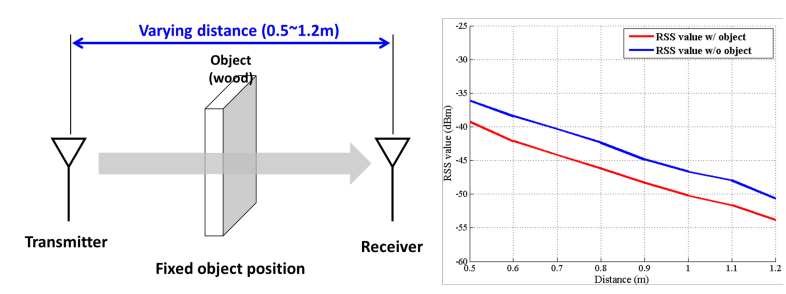

(b) Case 2: Fixed relative position with varying distance

Figure 10. Experiment results for additional EM waves attenuation due to the penetration effect. Each results shows a constant EM waves attenuation than open space case regardless of positions and distance between antennas.

Three experiment results are shown in Fig. 10. These experimental results show that additional power attenuation by penetration has almost constant values regardless of the antenna position, distance and object relative position. 


\subsection{Conclusion of underwater EM waves interference near objects}

The underwater EM waves interference near objects showed different characteristic with the air condition. In particular, the input impedance value had similar to an input impedance in open environment despite it belong to the reactive near field, and the RSS of EM waves had a few multi-path effect when the gap of propagation line and object is greater than radius of 4th Fresnel zone. It caused that water medium has short wavelength compared with air medium at same frequency, and the water medium considered as the lossy medium: the multi-path effects become extinct due to the large signal attenuation along to the additional travel distance. On the other hand, the additional loss due to the object penetration showed the specific and uniform attenuation characteristic regardless of distance and position when whole of 4th Fresnel zone belong to the object. Therefore, the penetration loss model along to the object characteristics is considered as EM waves distance-attenuation model in structured environments.

\section{Derivation of Underwater Range Sensor Model in Structured Environments}

\subsection{EM waves penetration loss model}

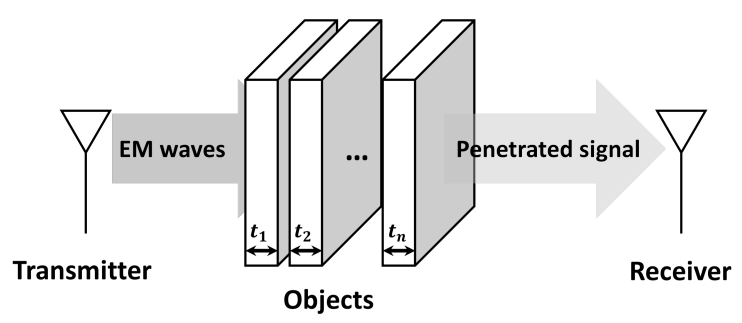

Figure 11. Influence factors of EM waves attenuation when EM waves penetrating objects. The penetration attenuation is affected by object depth and material type.

When an object exists between the transmitter antenna and the receiver antenna, and whole of 4th Fresnel zone belong to the object, it can be supposed that EM waves have additional loss due to the penetration. This penetration loss can be described as equation of object depth and object number as shown below as shown in Fig. $11[16,17]$ :

$$
L_{o b j}=\beta n+\gamma t_{m} \quad[\mathrm{~dB}] .
$$

$L_{o b j}$ is intended to capture the additional attenuation due to $n$ object with total object thickness $t_{m}=t_{1}+t_{2}+$ $\cdots+t_{n}$, located between the transmitter and the receiver. The first of the two calibration factors, $\beta$, is given in $\mathrm{dB}$ per object and represents the additional attenuation caused by penetration. The second calibration factor, $\gamma$, is given in $\mathrm{dB}$ per meter and represents the attenuation factor by material.

By adding the penetration loss model that only considers the penetration into the underwater sensor model for EM waves attenuation, the sensor model can estimate the transmitter-receiver separation robustly in an structure included environment. Subtracting Eq. (12) from Eq. (6), it results as:

$$
R S S=-20 \log _{10} R-20 R \alpha \log _{10} e+C-\beta n-\gamma t_{m}
$$

\subsection{Calibration factor experiments}

Two experiments were carried out to develop and verify the improved underwater sensor model. In this section, the RSS values in various structure materials were measured to determine the calibration factors $\beta$ and $\gamma$ depending on the material. 
Table 2. Calibration Factor Experiment Conditions

\begin{tabular}{cccccccc}
\hline & $n$ & $t_{m}[\mathrm{~m}]$ & $L_{o b j}$ & & $n$ & $t_{m}[\mathrm{~m}]$ & $L_{o b j}$ \\
\hline \multirow{4}{*}{ Wood } & 1 & 0.015 & 2.0906 & 1 & 0.02 & 2.9960 \\
& 1 & 0.024 & 2.9292 & & 1 & 0.03 & 3.4173 \\
& 2 & 0.030 & 4.0958 & Stone & 2 & 0.04 & 6.5826 \\
& 2 & 0.039 & 5.1298 & & 2 & 0.05 & 6.9262 \\
& 2 & 0.048 & 5.9621 & & 2 & 0.06 & 7.3574 \\
\hline \multirow{3}{*}{ Acrylic } & 1 & 0.005 & 2.3662 & & 1 & 0.002 & 43.4537 \\
& 2 & 0.007 & 5.7280 & & & & \\
& 2 & 0.010 & 6.8202 & & & 0.007 & 43.5414 \\
& & & & & & & \\
\hline
\end{tabular}

The calibration factors are determined based on the material of the objects. Because most of the underwater structures and the facilities consist of stone, wood and steel, the calibration factor experiment were conducted using these materials.

\subsubsection{Condition and procedure}

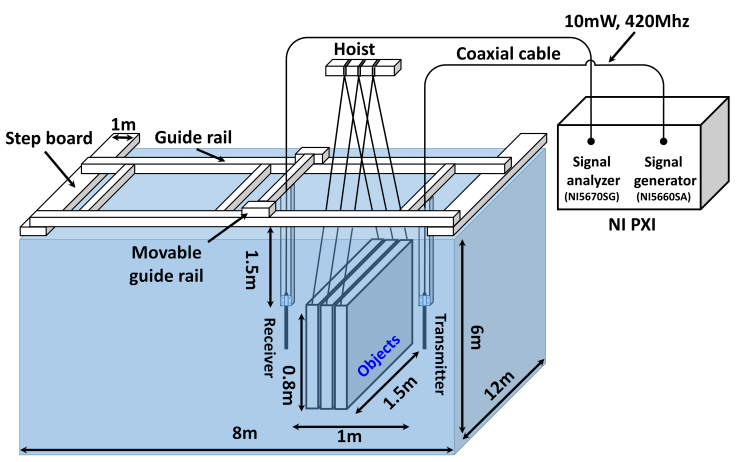

Figure 12. Schematic diagram of material calibration factor experiments.

The experimental environment is shown in Fig. 13. The object was $1.5 \mathrm{~m}$ wide, $0.8 \mathrm{~m}$ long with various thickness. The objects were deployed between antennas, and they were hung on two hoists. The distance between the nodes was $1 \mathrm{~m}$.

The experiment was performed as follows. First, the RSS values without object $S_{w / o}$ were measured. And then these experiments were repeated according to the various objects and thickness $S_{w / \text {. The material types }}$ were wood, stone and steel. When $n$ was greater than 1 , the gap between objects was kept a $5 \mathrm{~mm}$ using support. $L_{o b s}$ could be solved using $S_{w / o}$ subtracted by $S_{w /}$. Finally, the $\beta$ and $\gamma$ values are calculated using least squares. The experiment conditions are shown in Table 3.

\subsection{Experiment Result}

The calibration factors according to the materials is shown in Table 3. In case of dielectric materials such as stone and wood, attenuation power $L_{o b j}$ is linearly decreased depending on the object $t_{m}$. Also, whenever object number is increased. In case of steel, since EM waves can not penetrate due to its conductivity, $L_{\text {steel }}$ was 


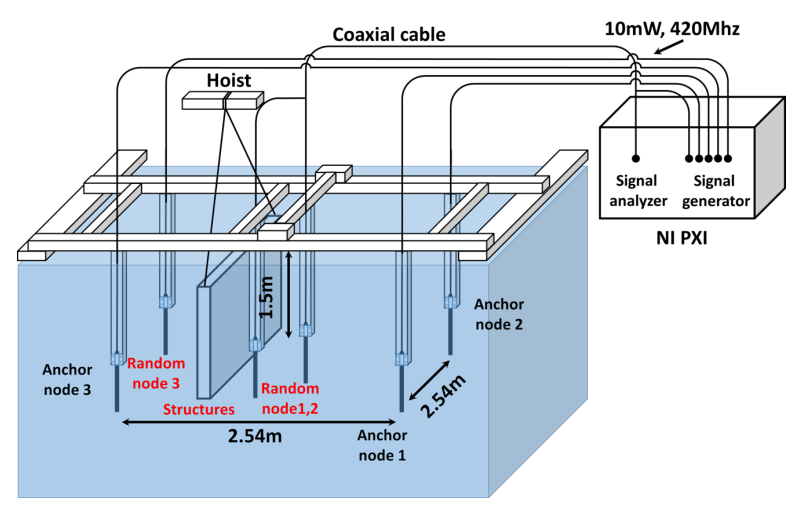

Figure 13. Schematic diagram of localization environment with structure. Anchor nodes were fixed near the edge of the test bed. The mobile node 1 and 2 estimated the their position using the received signal, and then they transmitted signal with their own frequency. Mobile node 3 estimated its position using the penetrated signal.

the largest value despite the small thickness. Thus, $L_{\text {steel }}$ can be considered as another distortion effect such as diffraction and reflection, and the effect of other distortions was trivial.

Table 3. Experiment Result for Calibration Factors

\begin{tabular}{c|l|l}
\hline & $\beta[\mathrm{dB} / \mathrm{n}]$ & $\gamma[\mathrm{dB} / \mathrm{m}]$ \\
\hline Wood & 0.6332 & 96.3408 \\
Stone & 2.3101 & 45.1200 \\
Acrylic & 1.4211 & 35.7411 \\
\hline \hline
\end{tabular}

\section{Localization Experiment in Environment with Underwater Structures}

The 2D localization experiment in infrastructure-based localization system was performed to verify the localization performance for structure environment.

\subsection{Experimental Condition and Procedure}

In order to check the penetration model performance, the mobile node localization was conducted in environment with object. The experimental environment was consisted of three anchor nodes (have specified localizations, and their position informations are known) and three mobile nodes (has randomized positions, and their positions are unknown) with $2.54 \mathrm{~m}$ long and $2.54 \mathrm{~m}$ wide square test bed as shown in Fig. 14 . The anchor nodes were fixed near the edge of the test bed, and their position information was known. The mobile nodes were located on the inner area of the test bed, and received signal from anchor nodes. The object was hung on the two hoists, and was located almost perpendicular to the mobile nodes. To check the node localization performance, all of the nodes were measured using a laser distance measuring instrument. It was assumed the EM waves signal becomes weaker due to the penetrating object only, and the object thickness and the material were known.

The experimental procedure was as follows: in order to verify the sensor model performance for non-object environment, the mobile nodes were estimated. The anchor nodes transmitted EM waves with different frequency bands, mobile nodes can know the identity of each anchor node. Nodes 1 and 2 received the signals from the anchor nodes, and estimated the distance using the sensor model, and then each node estimated theirs positions. Next, to verify the sensor model performance for an environment with the structures, mobile node 3 was estimated. Mobile node 1 and 2 broadcast their own estimated positions on different frequency bands. Mobile node 3 received the signals from the two mobile nodes, and then estimated its own position. In order 


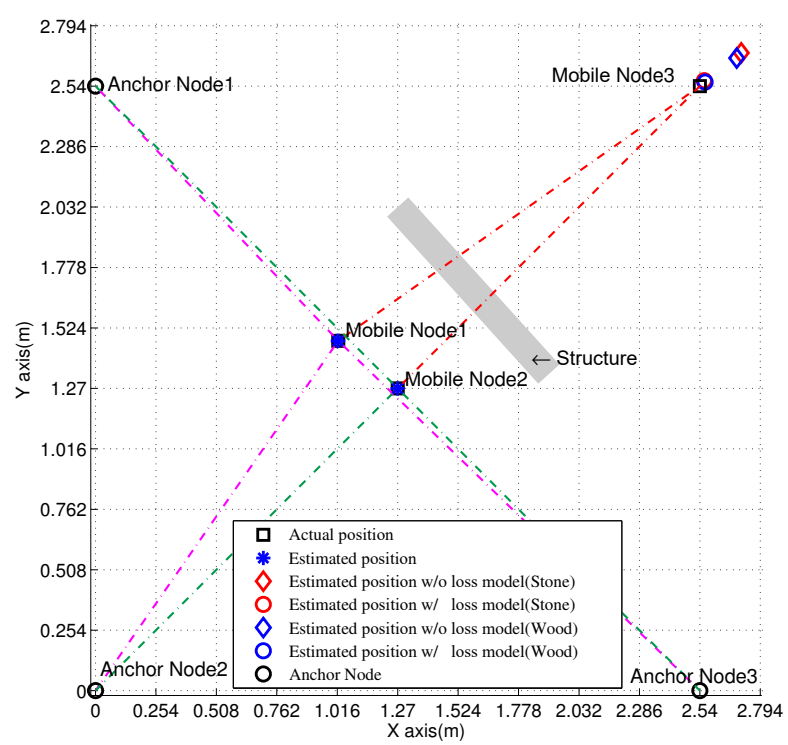

Figure 14. Mobile node localization condition and result

243 to check the penetration loss model performance, a different sensor model was used. One is the sensor model without additional loss factor for objects, the other is the sensor model with additional loss factor.

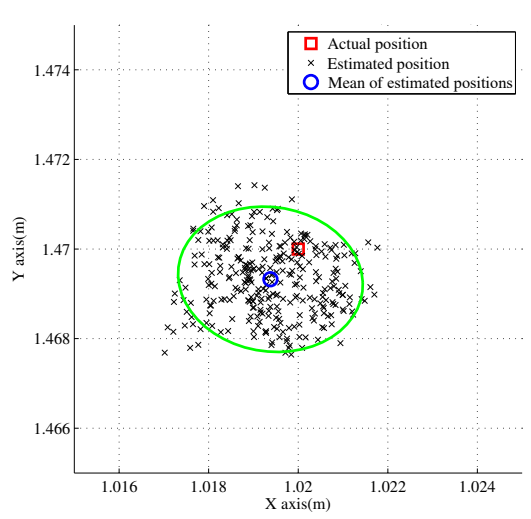

(a) Mobile node 1

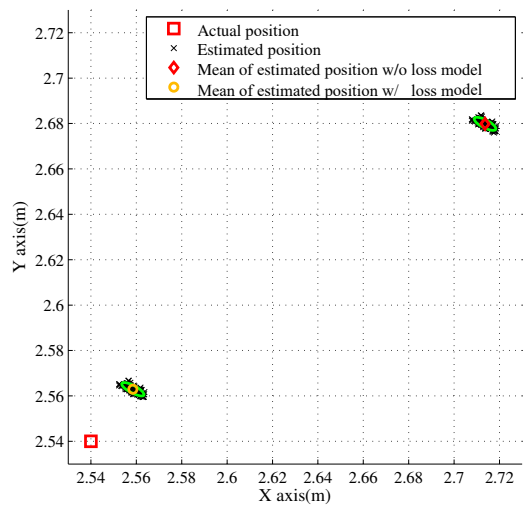

(c) Mobile node 3 with structure (Stone)

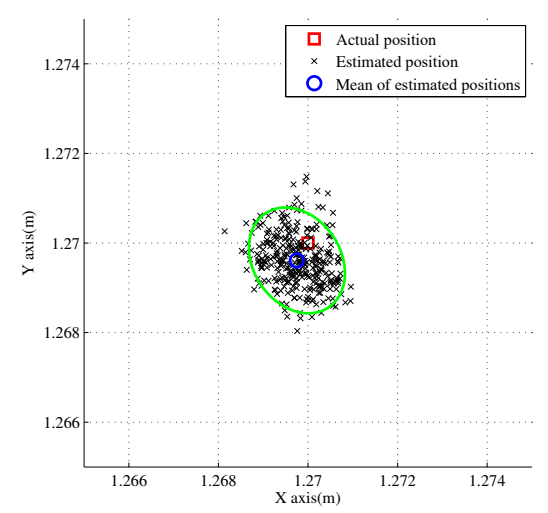

(b) Mobile node 2

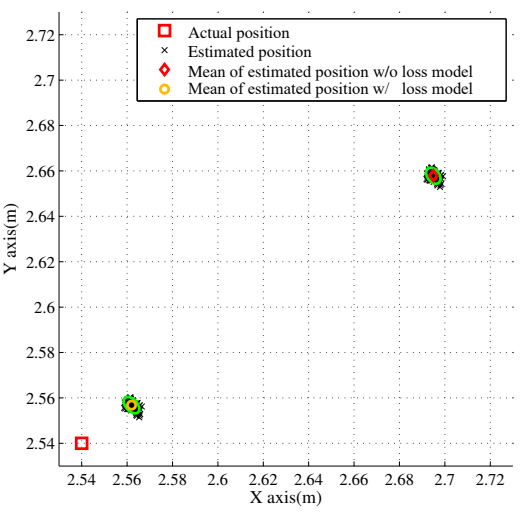

(d) Mobile node 3 with structure (Wood)

Figure 15. Mobile node localization results 
Table 4. Mobile Node Localization Conditions and Results

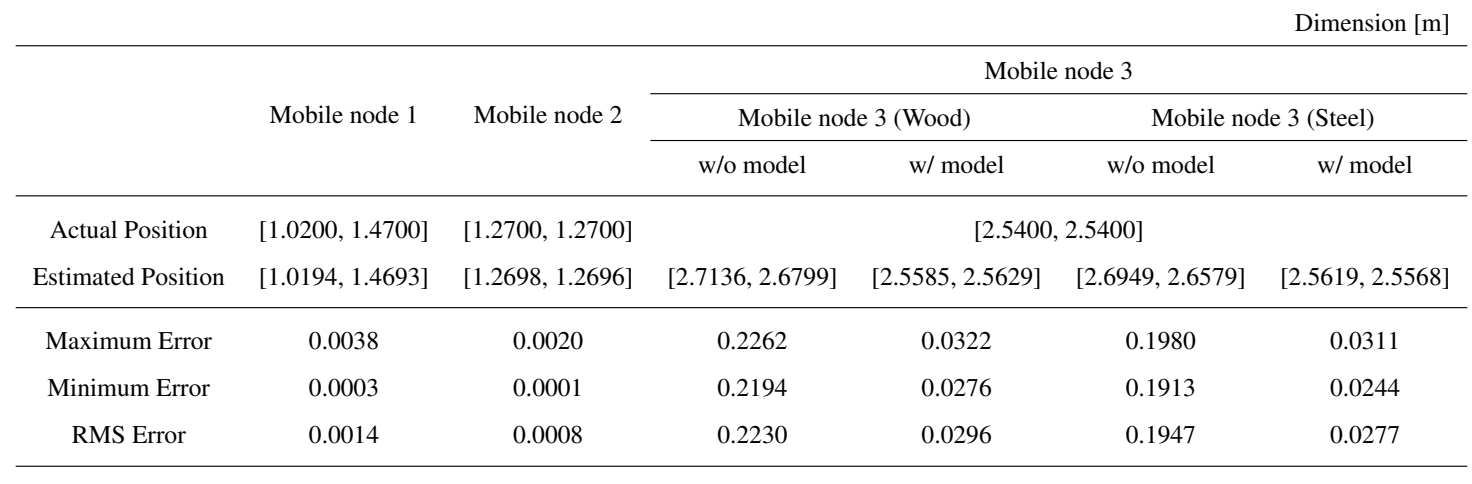

\subsection{Localization Result}

The localization results are shown in Fig. 14 and Fig. 15. First, the localization results of mobile node 1 and 2 were shown in Fig. 15(a) and Fig. 15(b). These figures show good position estimation results, and the estimated positions were inside of the covariance ellipse with a small error. It means that the localization result had good performance for non-object environments. The localization performances according to the structure material are shown in Fig. 15(c) (stone) and Fig. 15(d) (wood). Regardless of using the penetration model, the localization results had a gap from actual position. This may be caused by additional distortion of EM waves or environmental effect. However, the performance of the sensor with the penetration model is significantly improved from that without the penetration loss model. In Table 4, the RMS errors were decreased by $90 \%$.

\section{Conclusion}

In this paper analyzed the characteristic of EM waves propagation in structured environment to identify the signal interference by the structure, and suggested the EM waves attenuation model considering the distance and penetration loss by the structure.

The near-field effect and multi-path effect in fresnel zone were considered as factors of influencing the propagation characteristics of EM waves. As the results of several experiments, the underwater EM waves interference near objects showed different characteristic with the air condition. In particular, the input impedance value had similar to an input impedance in open environment despite it belong to the reactive near field, and the RSS of EM waves had a few multi-path effect when the gap of propagation line and object is greater than radius of 4th Fresnel zone. Also, the additional loss due to the object penetration showed the specific and uniform attenuation characteristic regardless of distance and position when whole of 4th Fresnel zone belong to the object.

Based on the EM waves propagation analysis, the object penetration loss was considered as EM waves additional loss model in structured environments. The proposed penetration loss model showed consistent and repeatable attenuation estimation capabilities. The underwater localizations in structured environment were conducted using proposed sensor model, and the showed the improved position estimation results with low biased error.

In the future, we will conduct more experiments with various materials and conditions, and will find the relation between EM waves propagation and object interference. Also we will prepare the structured environments localization in real sea condition.

1. Paull, L.; Saeedi, S.; Seto, M.; Li, H. AUV navigation and localization: A review. IEEE Journal of Oceanic Engineering 2014, 39, 131-149.

2. Marani, G.; Choi, S. Underwater target localization. IEEE Robotics \& Automation Magazine 2010, 1, 64-70.

3. Tuna, G.; Gungor, V.C. A survey on deployment techniques, localization algorithms, and research challenges for underwater acoustic sensor networks. International Journal of Communication Systems 2017. 
4. Li, J.; Lee, M.; Kim, J.; Park, S.; Park, S.; Suh, J. Guidance and control of P-SURO II hybrid AUV. 2012 Oceans-Yeosu. IEEE, 2012, pp. 1-7.

5. Akyildiz, I.F.; Pompili, D.; Melodia, T. Underwater acoustic sensor networks: research challenges. Ad hoc networks 2005, 3, 257-279.

6. Gordon, J.; Moscrop, A. Underwater noise pollution and its significance for whales and dolphins. The conservation of whales and dolphins: science and practice. John Wiley \& Sons, Chichester, United Kingdom 1996, pp. 281-319.

7. Shin, S.Y.; Namgung, J.I.; Park, S.H. SBMAC: Smart blocking MAC mechanism for variable UW-ASN (underwater acoustic sensor network) environment. Sensors 2010, 10, 501-525.

8. Park, D.; Kwak, K.; Chung, W.K.; Kim, J. Development of Underwater Short-Range Sensor Using Electromagnetic Wave Attenuation. IEEE Journal of Oceanic Engineering 2016, 41, 318-325. doi:10.1109/JOE.2015.2433851.

9. Park, D.; Kwak, K.; Kim, J.; Chung, W.K. 3D underwater localization scheme using EM wave attenuation with a depth sensor. 2016 IEEE International Conference on Robotics and Automation (ICRA), 2016, pp. 2631-2636. doi:10.1109/ICRA.2016.7487422.

10. Park, D.; Kwak, K.; Kim, J.; Chung, W.K. Underwater sensor network using received signal strength of electromagnetic waves. 2015 IEEE/RSJ International Conference on Intelligent Robots and Systems (IROS), 2015, pp. 1052-1057. doi:10.1109/IROS.2015.7353500.

11. Park, D.; Kwak, K.; Chung, W.K.; Kim, J. Development of underwater distance sensor using EM wave attenuation. Robotics and Automation (ICRA), 2013 IEEE International Conference on. IEEE, 2013, pp. 5125-5130.

12. Friis, H.T. Introduction to radio and radio antennas. Spectrum, IEEE 1971, 8, 55-61.

13. Ulaby, F.T.; Ravaioli, U.; Michielssen, E. Fundamentals of applied electromagnetics; Prentice Hall, 2014.

14. Freeman, R.L. Radio system design for telecommunication; Vol. 98, John Wiley \& Sons, 2006.

15. Balanis, C. Antenna Theory Analysis and Design 3rd edition, A John Wiley \& Sons. Inc., Publication 2005.

16. Ahmed, S.; Karmakar, G.C.; Kamruzzaman, J. An environment-aware mobility model for wireless ad hoc network. Computer Networks 2010, 54, 1470-1489.

17. Sommer, C.; Eckhoff, D.; German, R.; Dressler, F. A computationally inexpensive empirical model of IEEE $802.11 \mathrm{p}$ radio shadowing in urban environments. Wireless On-Demand Network Systems and Services (WONS), 2011 Eighth International Conference on. IEEE, 2011, pp. 84-90. 